\title{
Congenital longitudinal melanonychia: a case report
}

\author{
Nina Skornšek ${ }^{\circledR}$, Tijana Orešič Barač², Pij Bogomir Marko²
}

\begin{abstract}
Congenital longitudinal melanonychia is rarely seen in Caucasians and poses a diagnostic dilemma. It is characterized by the presence of hyperpigmented brown or black streaks visible in the nail plate. We present the case of 20-month-old boy with congenital longitudinal melanonychia, the diagnostic procedure, and management. Dermatoscopy of the nail revealed dark brown linear hyperpigmented lines in the nail plate with an irregular color. The diagnosis of a congenital nail matrix nevus was established on clinical grounds. A wait-and-see policy was taken. A biopsy was not performed because the cause of longitudinal melanonychia is essentially benign, especially in the congenital form. Subungual melanomas are very rare and mostly appear in middle-aged patients. Clinical, dermatoscopic, and histopathological guidelines that could directly distinguish between a benign nail matrix nevus and malignant subungual melanoma in the pediatric population have not been confirmed yet. The diagnostic dilemma therefore remains open.
\end{abstract}

Keywords: longitudinal melanonychia, congenital, childhood, nail pigment, pigmented nevus

Received: 10 July 2017 | Returned for modification: 27 August 2017 | Accepted: 2 September 2017

\section{Introduction}

Longitudinal melanonychia (LM) is a clinical condition characterized by vertical linear brown or black hyperpigmented streaks visible in the nail plate (1). It can be congenital or acquired. LM in children under 12 is uncommon in any race and it is especially rarely seen in Caucasians (3). The most common location for LM is on the thumb and third finger. Involvement of the toes is exceptional $(2,3)$. The condition is caused by increased production of melanin in the nail matrix, which is later deposited in the nail plate (4). Melanocytes are melanin-producing cells located in the epidermis, but they are not equally distributed in the human body. The density of melanocytes in the skin is approximately $1,150 / \mathrm{mm}^{2}$, and in the nail matrix the number is significantly lower, only $200 /$ $\mathrm{mm}^{2}$. Generally, the melanocytes in the proximal nail matrix do not actively produce melanin; however, around 50\% of the melanocytes in the distal nail matrix do. Even fewer melanocytes are located in the nail bed; they are either not present at all, or up to $50 / \mathrm{mm}^{2}$ and do not actively produce melanin. This explains why subungual melanoma in the nail bed epithelium usually turns out to be amelanotic and consequently a diagnostic challenge (5). Histologically, LM can be caused by focal melanocyte activation, melanocyte hyperplasia, a nail matrix (lentigo) nevus, or nail matrix (malignant) melanoma (4). Clinical differentiation between the potential causes of LM still poses a great diagnostic challenge because clinical findings can resemble those seen in malignant lesions, and therefore a pathological study is sometimes required. Congenital LM is usually benign and is mostly caused by a nail matrix nevus. Not many cases of subungual malignant melanoma in the pediatric population have been reported because the condition is very uncommon. Most of the published case reports have involved highly pigmented and Asian races (6).

\section{Case report}

A 20-month-old boy with skin phototype 2 (blond hair and blue eyes) presented with a nail streak of LM clearly visible on his third left toe. His parents stated that the lesion was congenital. The boy was generally healthy, without any chronic illnesses. He did not have any other melanocytic skin nevi. Malignant melanoma was not present in the family history. The history of trauma was negative. Examination revealed brown lines forming a streak in the medial $30 \%$ of the nail plate. No distal splitting of the nail was visible (Fig. 1). Dermatoscopic examination of the nail displayed dark brown linear lines of hyperpigmentation, approximately $4 \mathrm{~mm}$ in width, with irregular color involving the entire length of the nail.

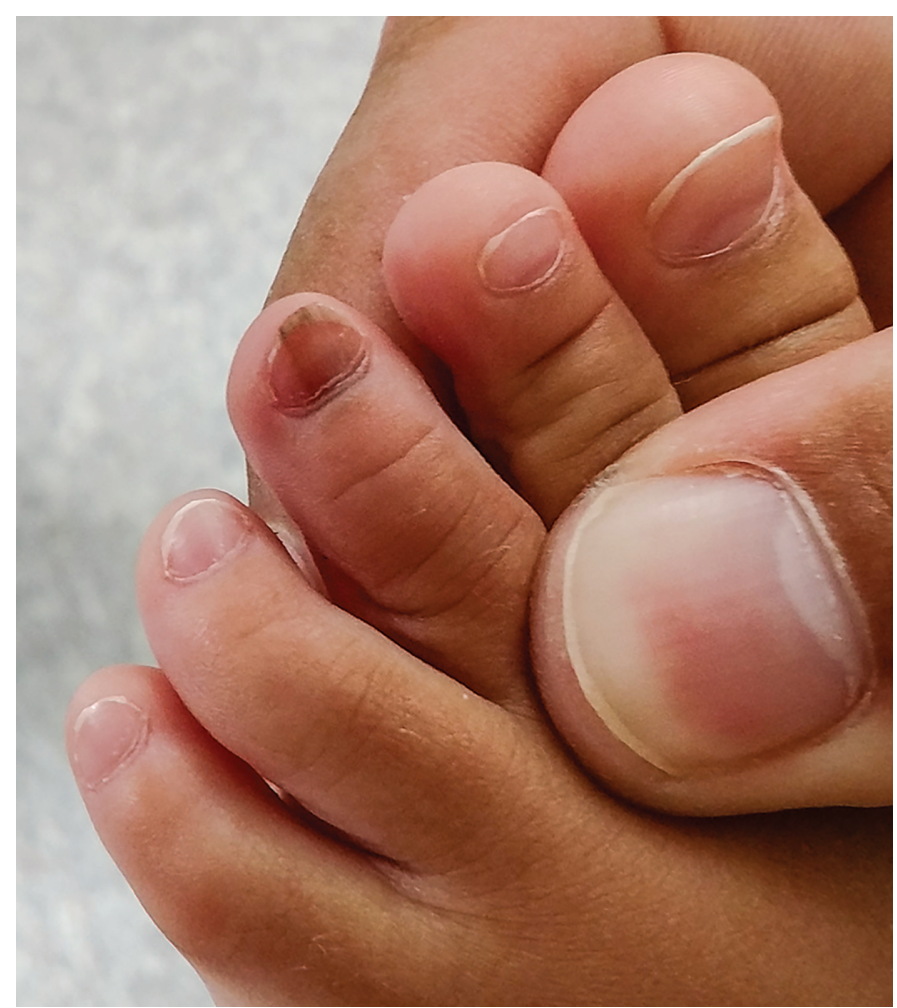

Figure 1 | The band of longitudinal melanonychia is visible on the third left toe. 
The darker brown discoloration was present on the proximal and distal part of the hyperpigmented band. The spacing between the lines was regular. There was no evident discoloration of the skin around the nail, although the hyperpigmented streak of LM was easily seen through the translucent cuticle; this condition is also known as pseudo-Hutchinson's sign (Fig. 2). The diagnosis of a congenital nail matrix nevus was established on clinical grounds. A wait-and-see policy was taken. One-year follow-up examinations were advised. The parents were informed to seek an immediate follow-up examination in the case of extension of the lesion, darkening color, or periungual involvement of the affected nail.

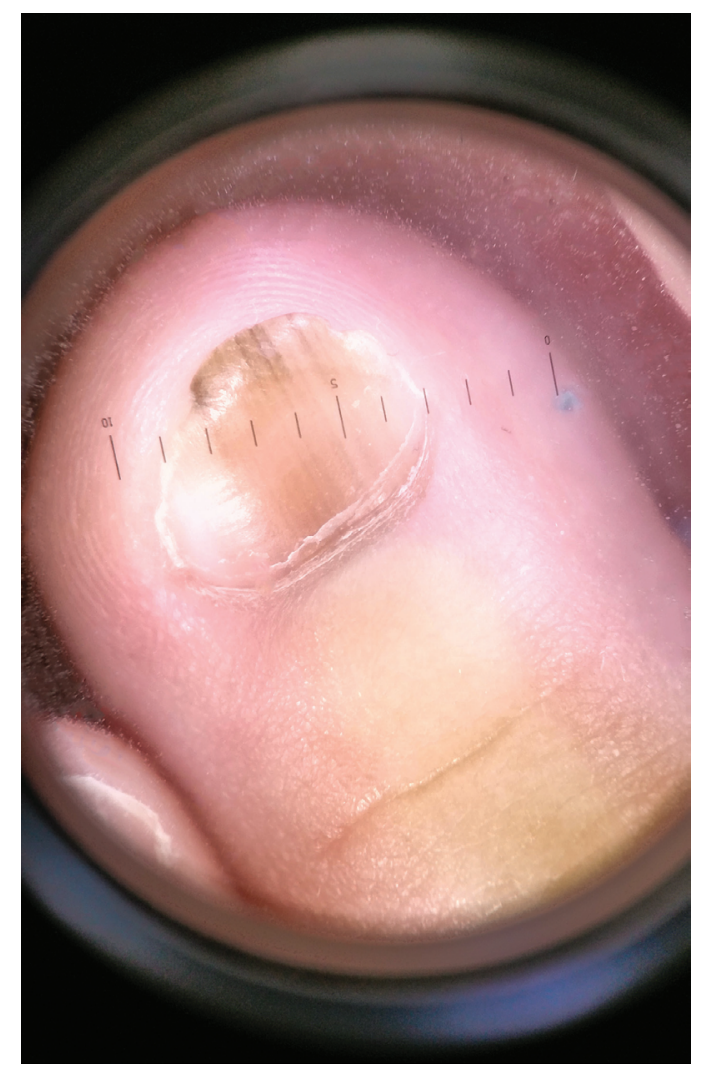

Figure 2 | Dermatoscopic presentation of longitudinal melanonychia.

\section{References}

1. Braun RP, Baran R, Le Gal FA, Dalle S, Ronger S, Pandolfi R, et al. Diagnosis and management of nail pigmentations. J Am Acad Dermatol. 2007;56:835-47.

2. Wong DE, Brodkin R, Rickeert R, McFalls SG. Congenital melanonychia. Int J Dermatol. 1991;30:278-80.

3. Leung AK, Robson WL, Liu EK, Kao CP, Fong JH, Leong AG, et al. Melanonychia striata in Chinese children and adults. Int I Dermatol. 2007;46:920-2.

4. Baran R, Kechijian P. Longitudinal melanonychia. Diagnosis and management. J Am Acad Dermatol. 1989;21:1165-75.

5. Ruben BS, Pigmented lesions of the nail unit: clinical and histopathologic features. Semin Cutan Med Surg. 2010;29:148-58.

6. Iorizzo M, Tosti A, Di Chiacchio N, Hirata SH, Misciali C, Michalany N, et al. Nail melanoma in children: differential diagnosis and management. Dermatol Surg. 2008;34:974-8.

7. Leaute-Labreze C, Bioulac-Sage P, Taieb A. Longitudinal melanonychia in children. Arch Dermatol. 1996;132:167-9.

\section{Discussion}

The main concern in the case presented was excluding subungual malignant melanoma. There are several diagnostic approaches to consider in the case presented. One of them is a wait-and-see policy, which was preferred in our case, and the second one is longitudinal excisional biopsy $(4,7,8)$. Biopsy and a histological examination is the only diagnostic method that can verify the diagnosis of a benign nail matrix nevus. In the case reported, a biopsy was not performed because excision of medial lesions in the proximal nail matrix can have an unacceptable outcome, resulting in irreversible nail dystrophy. Furthermore, the biopsy sample is only indicative of a minor segment of the nevus, and its interpretation can be deceiving (9). The most important reason to decide against it was the fact, supported by most published cases, that LM is essentially benign, especially in the congenital form. Less than $0.5 \%$ of malignant melanomas are present in prepubertal children (10). Subungual malignant melanoma is extremely uncommon and mostly appears in middle-aged patients over age 50 (9). In very young Caucasian children, a biopsy is not always necessary because the characteristics of the nevus can be predicted on clinical basis (11). The signs cited as indicative of early subungual melanoma are a hyperpigmented streak wider than 6 $\mathrm{mm}$, dystrophic changes of the nail, its onset after middle age, and the expansion of dark pigmentation from the band of LM into the adjoining cuticle and proximal or lateral nailfolds (Hutchinson's sign). Hutchinson's sign by itself is not pathognomonic, but it is an especially valuable predictor of malignant subungual melanoma (12). None of these factors were present in our case. To our knowledge, reports of congenital LM with absent signs of malignancy transforming into an invasive melanoma during childhood have not been reported yet. A malignant transformation in adult life is still possible, but is highly unlikely (13). Regular 1-year follow-up examinations are therefore required. It is necessary to stress the importance of an immediate examination in the case of expansion, change in color, or periungual involvement of the LM. Parents should also educate children with LM to watch for any alteration of the lesion throughout their entire lives.
8. Tosti A, Piraccini BM, Cagalli A, Haneke E. In situ melanoma of the nail unit in children: report of two cases in fair-skinned Caucasian children. Pediatr Dermatol. 2012;29:79-83.

9. Rigby HS, Briggs JC. Subungual melanoma: a clinico-pathological study of 24 cases. Br J Plast Surg. 1992;45:275-8.

10. Roth ME, Grant-Kels JM, Kuhn K, Greenberg RD, Hurwitz S. Melanoma in children. J Am Acad Dermatol. 1990;22:265-74.

11. Baran R, Kehecijan P. Longitudinal melanonychia (melanonychia striata): diagnosis and management. J Am Acad Dermatol. 1989;21:1165-75.

12. Saida T, Ohshima Y. Clinical and histopathologic characteristics of early lesions of subungual malignant melanoma. Cancer. 1989;63:556-60.

13. Goettmann-Bonvallot S, Andre J, Belaich S. Longitudinal melanonychia in children: a clinical and histopathologic study of 40 cases. J Am Acad Dermatol. 1999;41:17-22. 